\title{
Spatiotemporal beam shaping in nonlinear multimode fibers
}

\author{
K. Krupa ${ }^{1}$, V. Couderc ${ }^{2}$, M. Fabert ${ }^{2}$, A. Tonello ${ }^{2}$, A. Barthélémy ${ }^{2}$, V. Kermene ${ }^{2}$, \\ A. Desfarges-Berthelemot ${ }^{2}$, G. Millot ${ }^{3}$, D. Modotto ${ }^{1}$, S. Wabnitz ${ }^{1,4}$ \\ ${ }^{1}$ Dipartimento di Ingegneria dell'Informazione, Università di Brescia, via Branze 38, 25123, Brescia, Italy \\ ${ }^{2}$ Université de Limoges, XLIM, UMR CNRS 7252, 123 Av. A. Thomas, 87060 Limoges, France \\ ${ }^{3}$ Université de Bourgogne Franche-Comté, ICB, UMR CNRS 6303, 9 Av. A. Savary, 21078 Dijon, France \\ ${ }^{4}$ Istituto Nazionale di Ottica del Consiglio Nazionale delle Ricerche (INO-CNR), Via Branze 45, 25123 Brescia, Italy \\ katarzyna.krupa@unibs.it
}

\begin{abstract}
Kerr beam self-cleaning in graded-index multimode fibers is accompanied by power-dependent temporal pulse reshaping. We explore the complex nonlinear dynamics with a single long pulse, where the optical power is continuously varied across its profile. (C) 2018 The Author(s)

OCIS codes: $190.4420,060.4370,190.3270$.
\end{abstract}

\section{Introduction}

The spatiotemporal nature of nonlinear mode coupling in graded index (GRIN) is responsible for the experimental observation of multimode solitons [1]. Work by Krupa et al. [2] has more recently shown that the Kerr effect in GRIN fibers may lead to highly stable beam self cleaning for sub-ns pulses. Temporal pulse compression with Kerr beam cleaning was already observed in the highly dispersive regime with fs pulses in Ref. [3]. In this work, we experimentally and numerically analyze the complex nonlinear evolution of self-cleaned beams in the temporal domain by direct detection of quasi-CW pulses in normal dispersion regime and when the dispersion length is much longer than the fiber length [4].

\section{Experimental and numerical results}

Our experiments were carried out with an amplified microchip Q-switched laser delivering $740 \mathrm{ps}$ pulses at a repetition rate of $27 \mathrm{kHz}$, with a central wavelength of $1064 \mathrm{~nm}$. The maximum energy per pulse can reach more than $100 \mu \mathrm{J}$, with a peak power level up to $150 \mathrm{~kW}$. The optical beam with $40 \mu \mathrm{m}$ diameter was coupled into a 12-m long GRIN 50/125 multimode silica fiber. A fast In-GaAs PIN photodiode was connected to a 20-GHz bandwidth oscilloscope and used to measure the output pulse envelope with samples spaced by $25 \mathrm{ps}$. The photodiode had an active area 150 times smaller than the area of the imaged near field, which was properly magnified. The experimental results when the photodiode was placed at the center of the near-field image are shown in panel (a) of Fig.1 . Upon increases of the
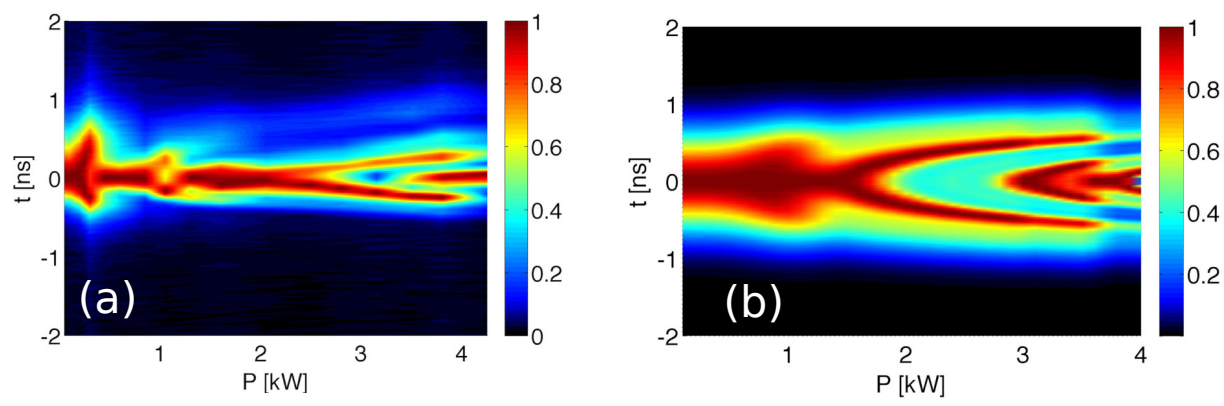

Fig. 1. Experimental results of normalized temporal profiles taken at the center of the output beam upon input coupled peak power (a). Numerical evolution for $1 \mathrm{~ns}$ pulse calculated from the transmission of the fundamental mode upon peak power after $12 \mathrm{~m}$ with 16 propagated modes (b).

input power, the temporal reshaping of the pulse envelope was observed to exhibit a complex behaviour, composed 
by a series of temporal broadenings, followed by the formation of a dip at the pulse center and then the growth of a narrower and high power peak. Since in our case the effect of dispersion is negligible, the long pulse can be modeled as a sequence of different instantaneous power values and the nonlinear evolution of the corresponding modal amplitudes $a_{n}(z)$ is described by the following standard equations:

$$
\frac{d a_{n}}{d z}=i \gamma \sum_{m, p, q} Q_{m, p, q, n} a_{m} a_{p}^{*} a_{q} \exp \left(i \Delta \beta_{m, p, q, n} z\right)
$$

$\Delta \beta_{m, p, q, n}=\beta_{m}-\beta_{p}+\beta_{q}-\beta_{n}$ is the phase mismatch for the mode group with indices $m, n, p, q$ and overlap coefficients $Q_{m, p, q, n}=\iint \psi_{m} \psi_{p}^{*} \psi_{q} \psi_{n}^{*} d x d y$, being $\psi_{n}(x, y)$ the $\mathrm{n}$-th element of the orthonormal basis of guided modes and $\gamma$ is the nonlinear coefficient of the fiber. From Eq. 1 it is possible to calculate the input-output transmission ratio of the
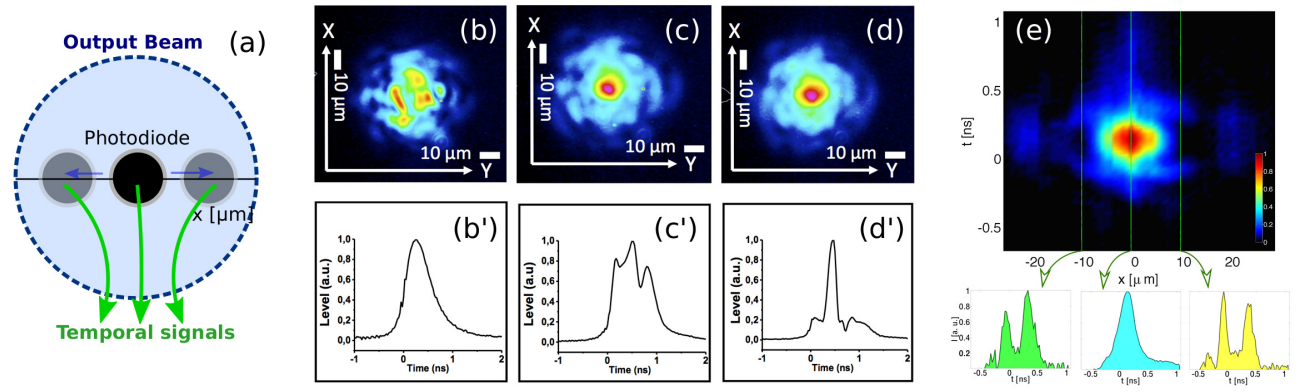

Fig. 2. Relative position of photodiode with respect to the output near-field image (a). Near field and temporal envelope in $x=0$ for $0.3 \mathrm{~kW}$ (b,b'), $2 \mathrm{~kW}$ (c,c'), $4 \mathrm{~kW}$ (d,d') coupled power. Temporal waveforms upon the photodiode position $x$ at $2 \mathrm{~kW}$ (e).

fundamental mode (whose intensity is located in the center of the beam) upon power. The numerical study (panel (b) of Fig.1) of the transmission into the fundamental mode upon peak power has features similar to experiments including the dip and the temporal narrowing. Additional experimental results are illustrated in Fig.2: panel (a) outlines the moving of the position of the photodiode across the output near-field image. Panels (b)-(d) show the progress of beam cleaning when increasing input beam power. Panels (b')-(d') show the corresponding temporal waveforms when the photodiode is placed at the center of the image $x=0$. Note that in panel (d') the temporal duration reduces to $175 \mathrm{ps}$. Panel (e) shows a collection of temporal waveforms (including some selected profiles) for different positions of the photodiode at $2 \mathrm{~kW}$. As can be seen the central part of the output beam is carried by a common temporal envelope. On the other hand temporal dips are observed when the photodiode is offset by $|x| \sim 10 \mu \mathrm{m}$ from the beam center, when the image is rescaled to the size of the fiber core.

In conclusion, we have shown experimentally that spatiotemporal mode coupling in nonlinear GRIN fibers may enable the simultaneous cleanup and temporal reshaping, which may result into a strong reduction of the pulse duration for a sub-ns pulse. These results may have important applications in the development of high power mode-locked fiber lasers based on multimode optical fibers.

We acknowledge support from: the European Research Council (ERC) under the European Union's Horizon 2020 research and innovation programme, grant No. 740355 and Marie-Sklodowska-Curie grant No. GA-2015-713694 (MULTIPLY); Horiba Medical and Leukos (MSCA MULTIPLY, BECLEAN project); iXcore research foundation; Labex ACTION program (contract ANR-11-LABX-0001-01).

\section{References}

1. W. H. Renninger and F. W. Wise, “Optical solitons in graded-index multimode fibres,” Nat. Commun. 11,1719 (2012).

2. K.Krupa, A.Tonello, B.M. Shalaby, M. Fabert, A. Barthélémy, G. Millot, S. Wabnitz, and V. Couderc, "Spatial beam self-cleaning in multimode fibre," Nat. Photon. 11, 234-241 (2017).

3. Z. Liu, L. G. Wright, D. N. Christodoulides and Frank W. Wise, "Kerr self-cleaning of femtosecond-pulsed beams in graded-index multimode fiber," Opt. Lett. 16, 3675-3678 (2016).

4. K. Krupa, A. Tonello, V. Couderc, A. Barthélémy, G. Millot, D. Modotto, S. Wabnitz, "Spatiotemporal light beam compression from complex nonlinear mode mixing," arXiv:1711.11478. 